\title{
THE EXTRACTION OF URBANIZED AREAS THROUGH IMAGES OF HIGH RESOLUTION NIGHTTIME LIGHTS
}

\author{
B. Arellano ${ }^{1}$, J. Roca ${ }^{1 *}$ \\ ${ }^{1}$ Technical University of Catalonia (blanca, josep)@upc.edu \\ ICWG III/7: Landuse and Landcover Change Detection
}

\begin{abstract}
KEY WORDS: Nighttime lights, DMSP-OLS, SNPP-VIIRS, Luojia 1-01, urban area, rural area, Barcelona Metropolitan Region, Shenzhen City
\end{abstract}

\begin{abstract}
:
Satellite nocturnal images of the earth are a useful way to identify urbanisation. Nighttime lights have been used in a variety of scientific contributions, including studies on the identification of metropolitan areas as well as landscapes impacted by urbanization. However, the study of urban systems by nighttime light imagery has had a fundamental limitation to date: the low spatial resolution of satellite sensors. Although the DMSP Operational Linescan System (OLS) has been gathering global low-light imaging data for over 40 years, its $2.7 \mathrm{~km}$ /pixel footprint has limited its use for in-depth studies of urban development. The 2011 launch by NASA and the NOAA of the Suomi National Polar Partnership (SNPP) satellite, with the Visible Infrared Imaging Radiometer Suite (VIIRS) sensor on board, has led to a significant improvement. This instrument has better spatial resolution (742 m/pixel), on-board calibration, a greater radiometric range, and fewer saturation and blooming problems than DMSP-OLS data. However, it still has considerable limitations for the in-depth study of the area and internal structure of urban systems.
\end{abstract}

The launch of Luojia 1-01 in June 2018 has increased expectations. LJ1-01 is a nano satellite that can obtain high-resolution nocturnal images (130 metres/pixel). The aim of this paper is to analyse, and compare with previous satellites, the new instrument's capacity to delimit the urbanised area and its efficiency in identifying types of urban landscape (compact, dispersed and rurban). The study cases are Barcelona Metropolitan Region (Spain) and Shenzhen City (China).

\section{INTRODUCTION}

Since the mid-twentieth century there has been a true "explosion" of urbanization on a global scale. The urban population has grown from 750 million people in 1950 to 2,860 in 2000 , more than $50 \%$ of the world population. In the Developed World, the urban model has suffered significant changes in recent decades, transforming from a model of urban continuum of medium and high densities to a model of an endless diffuse and sprawled city, driven by technological innovation processes, separation of functions and seeking proximity to nature. Therefore, since 1950 there has been a real reversal in the topology of the landscape. The process of urban sprawl has relegated to open spaces the role of auxiliary elements within the spatial structure. The sprawl in residential areas is linked to the gradual decentralisation of economic activity, first the industry, and then the services and even the most qualified tertiary activities. Urban sprawl, the massive consumption of land, can be found worldwide, although it takes many different forms in different regions and continents.

Satellite nocturnal images of the earth are a useful way to identify urbanisation (Elvidge et alt, 2001). Nighttime lights have been used in a range of scientific contributions, including studies the identification of megalopolises (Florida et alt., 2008) and urban landscapes (Arellano \& Roca, 2016). This paper assumes that night-time lights satellite imagery provides valuable information for the identification of human landscapes, such as rural and urbanized areas. The "dark" landscapes are certainly related to rural settlements. The landscapes of light and darkness detect more clearly than traditional statistics based on the percentage of urban/rural forms of human settlement on the world population, with the advantage, in turn, of allow it to be studied on a subnational level, which is not possible when simply using official statistics. Concerning the "lit" landscapes, it clearly identifies areas of the world characterized by high human artificialisation. The electricity supply, along with the division of land into plots and the "lines" of the streets, represent the first steps in the process of urbanisation. The almost universal access to electrical energy as well as the diverse intensity of its use makes the analysis of night-time images an exceptional tool for studying the urbanisation gradient on a world scale.

However, the study of the extent and internal structure of urban systems by nighttime light imagery has had a fundamental limitation to date: the low spatial resolution of satellite sensors. Although the DMSP Operational Linescan System (OLS) has been gathering global low-light imaging data for over 40 years, its $2.7 \mathrm{~km} /$ pixel footprint has limited its use for in-depth studies of urban development. The 2011 launch by NASA and the NOAA of the Suomi National Polar Partnership (SNPP) satellite, with the Visible Infrared Imaging Radiometer Suite (VIIRS) sensor on board, has led to a significant improvement. This instrument has better spatial resolution (742 m/pixel), onboard calibration, a greater radiometric range, and fewer saturation and blooming problems than DMSP-OLS data (Elvidge et alt., 2013). However, it still has considerable limitations for the in-depth study of the extent and internal structure of urban systems. The launch of Luojia 1-01 in June 2018 has increased expectations (Jiang et alt., 2018). LJ1-01 can obtain high-resolution nocturnal images (130 meters/pixel),

\footnotetext{
* Corresponding author
} 
with a dynamic range above 14 bits at night and a spectrum rate of $0.46-0.98 \mu \mathrm{m}$.

The aim of this paper is to analyse, and compare with previous satellites, the new instrument's capacity to delimit the urbanized area and its efficiency in identifying types of urbanized landscapes (compact, dispersed and rurban).

The study cases are Barcelona Metropolitan Area (MRB, 3,200 $\mathrm{km}^{2}, 4.7$ million inhabitants) and Shenzhen City $\left(1,997.47 \mathrm{~km}^{2}\right.$, 13.03 million inhabitants). The methodology used consisted of developing a series of models showing whether land has been urbanized according to information provided by Corine Land Cover (CLC), in the case of Barcelona, and Finer Resolution Observation - Global Land Cover (FROM-GLC), in the case of Shenzhen, by means of logistic regression, with the intensity of nighttime illumination provided by DMSP-OLS, Black Marble, SNPP-VIIRS and Luojia 1-01 as an independent variable. Figures 1 (Barcelona) and 2 (Shenzhen) show the studied areas.
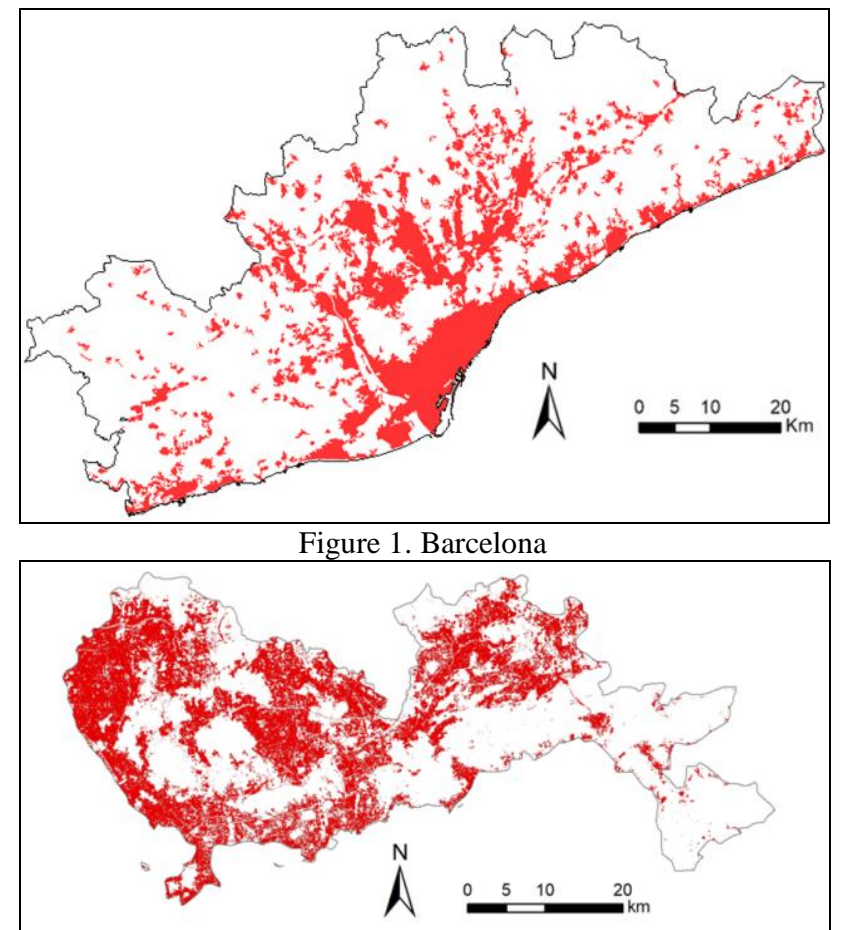

Figure 2. Shenzhen

\section{METHODOLOGY}

The methodology used in this paper has consisted:

- Build a file in which each register is a pixel of the highest resolution image (Luijoa-01) (200,568 in the MRB, 110,653 in Shenzhen), incorporating the night lights of the 4 sensors (DMSP-OLS, Black Marble, VIIRS and Luijoa$01)$.

- In the case of Luojia1-01 images, the DN (Digital Number) values of the are converted to radiance values using the following equation, based on laboratory calibration provided by the satellite data website:

$$
\boldsymbol{r}=\mathrm{DN}^{3 / 2} \cdot 10^{-10} \cdot \boldsymbol{w}
$$

Where $r$ is the radiance value, DN is the digital number obtained by Luojia1-01, and $\mathrm{w}$ is the bandwidth, whose unit is $\mathrm{Wm}^{-2} \mathrm{sr}^{-1}$. The radiometric range of Luojia1-01 is $0.46-0.98 \mu \mathrm{m}$, so that $\mathrm{w}$ is equal to $0.52 \mu \mathrm{m}$.
- In the rest of the sensors, the logarithmic transformation of the digital numbers ( $\mathrm{Yu}$ et al, 2018) has been used as independent variables in the logistic regression models.

- Identify urbanized areas through CLC and FROM-GLC (rural $=0$; $\operatorname{urban}=1$ ).

- Below is a set of logistic regressions (a model for each of the images analyzed) with urbanization (1-0) as a dependent variable and the intensity of the night light supplied by the different sensors as independent variables.

- Once the logistic regression is carried out, analyze the \% of successes, especially in the urbanized land, as well as other indicators of the goodness of the adjustment achieved.

- Select all the urbanized land pixels as well as the rural ones correctly classified in the previous logistic regression, and, with that selection perform another logistic regression, repeating iteratively, until the $\%$ of urbanized land successes do not improve meaningfully.

- Once the process is finished, it is necessary to "recover" the rural pixels discarded in the previous stages (due to being poorly classified) as well as calculate the cross table with all the pixels, definitively establishing the $\%$ of successes in the urbanized land and the rural land (overall accuracy). Check the statistical significance, as well as the "measure of agreement" Kappa, estimating the validity of each model developed.

- Repeat the above procedure for all sensors.

The methodology developed allows to evaluate the utility of DMSP-OLS, Black Marble, SNPP-VIIRS and Luojia 1-01 to estimate the extent of urbanization in the MRB and Shenzhen City, compare the results obtained by each sensor and check the degree of improvement achieved by Luojia 1-01.

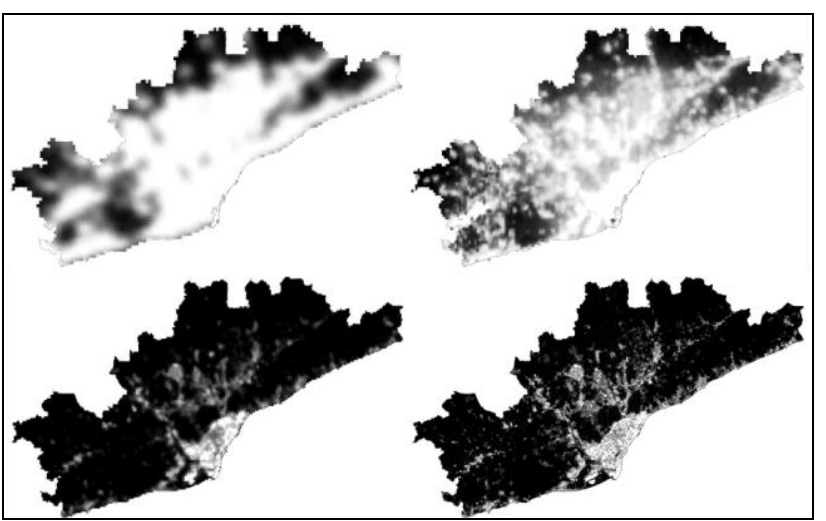

Figure 3. Night lights obtained by DMSP-OLS, Black Marble, SNPP-VIIRS and Luojia 1-01 (MRB)

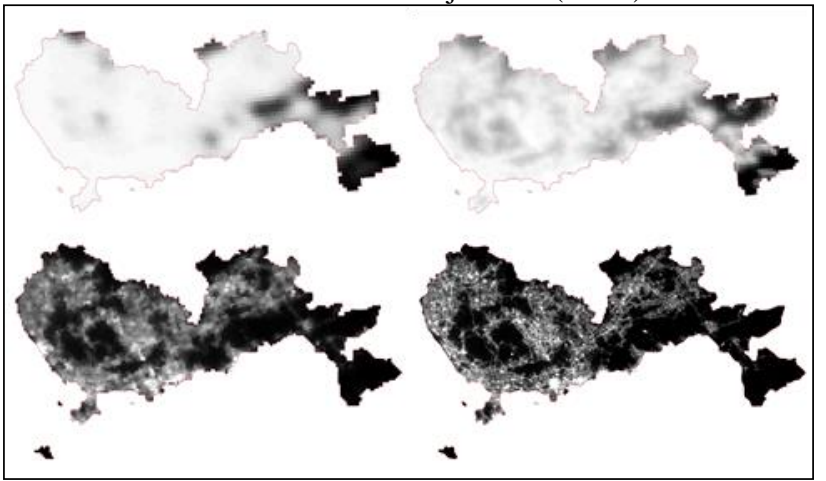

Figure 4. DMSP-OLS, Black Marble, SNPP-VIIRS and Luojia 1-01 (Shenzhen) 


\section{RESULTS}

\subsection{Metropolitan Region of Barcelona (MRB)}

Figures 5 to 8 show DPSP-OLS (2012), Black Marble (2012), SNPP-VIIRS (2016) and Luojia 1-01 (2018) nighttime lights and the urbanized land according to CLC (2012) in MRB. Tables 1 to 3 show the synthetic results (overall accuracy) of the iterative logistic regressions obtained for these last three sensors, since it has not been possible to obtain stable results with DMSP-OLS.

Despite the good results highlighted in the preceding literature about the capabilities of DMSP-OLS to identify urban development (Su et alt., 2015; Zhou et alt., 2014, the proposed methodology fails as logistic regressions not able to converge due to the excessive confusion of the pixels existing in the study area (MRB). Given the low depth (0-63) as well as the very high footprint of the pixel $(2.7 \mathrm{~km} / \mathrm{pixel})$, the confusion between lit and dark pixels is pronounced, decreasing the usefulness of the information provided by DMSP-OLS to analyse in detail the urbanization process, especially in a relatively small area such as the MBR. The high level of brightness (44.45 in the scale 0-63), superior to the cut-off used in other studies (Arellano \& Roca, 2018) to identify urbanized areas (37 on 63), explains the inability of the logistic regression model to converge in the area of study.

Adopting the aforementioned cut-off of 37, DMSP-OLS obtains a success level of $96.42 \%$ in the case of urbanized covers, but fails at $57.04 \%$ in the case of rural pixels, obtaining an overall accuracy of only $54.89 \%$. Consequently, the Kappa Value obtained is very small (0.232). However, said cut-off has been adjusted for the "urbanized areas" defined by the US Census Bureau (USCS, 2010), very different from the urbanized land covers of the CLC (CLC, 2019), by incorporating large undeveloped areas.

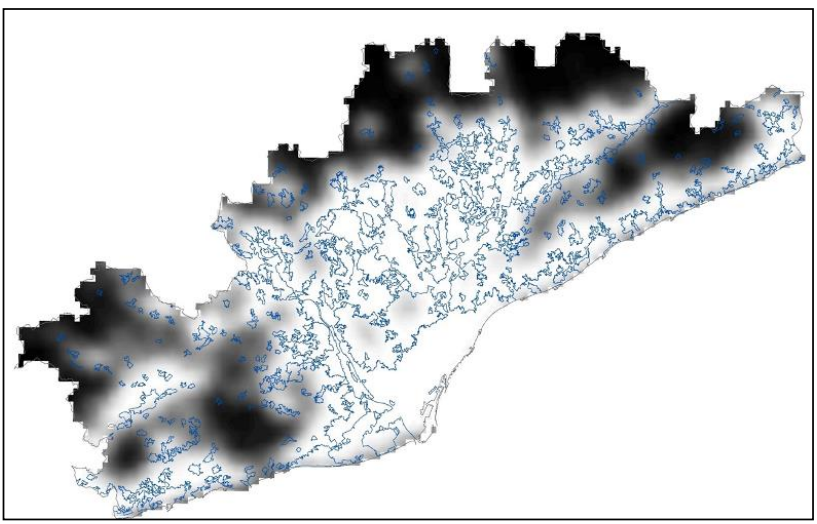

Figure 5. DMSP-OLS (MRB)

In the case of Black Marble (NASA, 2012), figure 6 and table $n$. 1 present the result of the logistic regression. The model converges at intensity 195 after 17 iterations, reaching $66.79 \%$ of hits in rural land, $91.28 \%$ in urbanized land and an overall accuracy of $72.25 \%$.

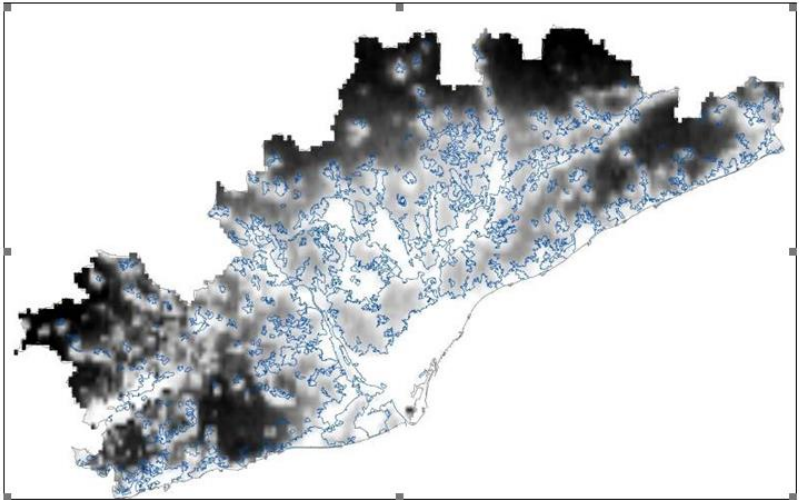

Figure 6. Black Marble (MRB)

\begin{tabular}{|ccc|ccc|}
\hline & & & \multicolumn{3}{|c|}{ Logistic Regression } \\
& & & Rural & Urbanized & Total \\
\hline \multirow{4}{*}{ Rural } & N. Pixels & 104080 & 51754 & 155834 \\
& & \% Row & $66,79 \%$ & $33,21 \%$ & $100,00 \%$ \\
& & $\%$ Column & $96,39 \%$ & $55,90 \%$ & $77,70 \%$ \\
\cline { 3 - 6 } CLC & \multirow{3}{*}{ Urbanized } & N. Pixels & 3903 & 40831 & 44734 \\
& & \% Row & $8,72 \%$ & $91,28 \%$ & $100,00 \%$ \\
& & \% Column & $3,61 \%$ & $44,10 \%$ & $22,30 \%$ \\
\cline { 3 - 6 } & \multirow{3}{*}{ Total } & N. Pixels & 107983 & 92585 & 200568 \\
& & \% Row & $53,84 \%$ & $46,16 \%$ & $100,00 \%$ \\
& & \% Column & $100,00 \%$ & $100,00 \%$ & $100,00 \%$ \\
\hline
\end{tabular}

Table 1. Urbanized Land (CLC) vs Black Marble (MRB)

Black Marble, with a pixel depth of 16 bits, and a spatial resolution of 0.0066667901 degrees (compared to 8 bits and 0.0083333333 of DMSP-OLS) obtains results that can be described as poor, as reflected by the Kappa coefficient (0.420).

Logistic regression models developed with SNPP-VIIRS (figure 7 and table 2) get better results. Logistic regressions converge after 21 iterations, obtaining $80.9 \%$ of hits in rural land and $88.3 \%$ in urbanized land. Overall accuracy reaches $82.52 \%$.

SNPP-VIIRS, with a pixel depth of 32 bits, and a spatial resolution of 0.0041666667 degrees, obtains medium-high results to identify the extent of artificialized covers of the MRB, as reflected by the Kappa coefficient (0.578).

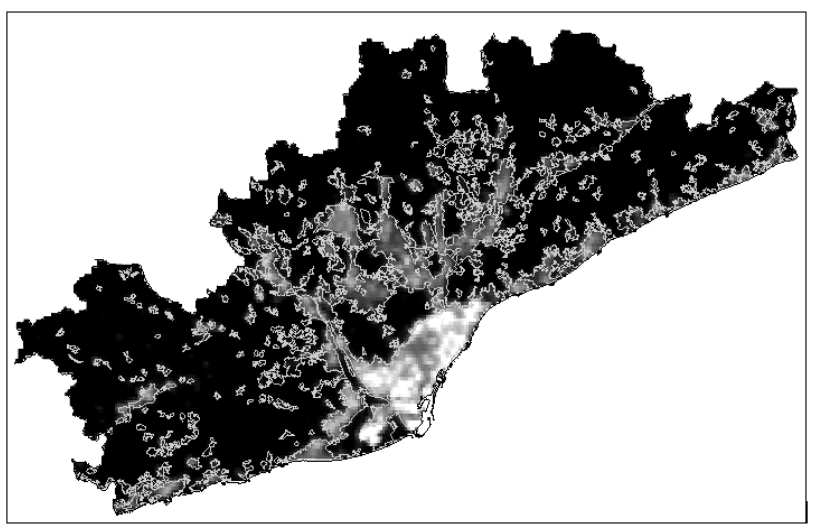

Figure 7. SNPP-VIIRS (MRB) 


\begin{tabular}{|ccc|ccc|}
\hline & & & \multicolumn{3}{|c|}{ Logistic Regression } \\
& & Rural & Urbanized & Total \\
\hline \multirow{4}{*}{ CLC } & \multirow{4}{*}{ Rural } & N. Pixels & 125994 & 29840 & 155834 \\
& & \% Row & $80,85 \%$ & $19,15 \%$ & $100,00 \%$ \\
& & $\%$ Column & $96,02 \%$ & $43,03 \%$ & $77,70 \%$ \\
\cline { 2 - 6 } & \multirow{3}{*}{ Urbanized } & N. Pixels & 5227 & 39507 & 44734 \\
& & \% Row & $11,68 \%$ & $88,32 \%$ & $100,00 \%$ \\
& & $\%$ Column & $3,98 \%$ & $56,97 \%$ & $22,30 \%$ \\
\cline { 2 - 6 } & \multirow{3}{*}{ Total } & N. Pixels & 131221 & 69347 & 200568 \\
& & \% Row & $65,42 \%$ & $34,58 \%$ & $100,00 \%$ \\
& & \% Column & $100,00 \%$ & $100,00 \%$ & $100,00 \%$ \\
\hline
\end{tabular}

Table 2. Urbanized Land (CLC) vs SNPP-VIIRS (MRB)

In the case of Luojia 1-01 (figure 8 and table 3), logistic regressions converge after 12 iterations, obtaining $87.8 \%$ of successes in rural land and $89.2 \%$ in urbanized land, with an overall accuracy of $88.08 \%$. The degree of validity of the night light intensity obtained by means of the Luojia 1-01 sensor (with a 32-bit pixel depth and a spatial resolution of 130 meters/pixel) can be considered high, as demonstrated by Kappa coefficient (0.691) achieved as well as the rest of the indicators used.

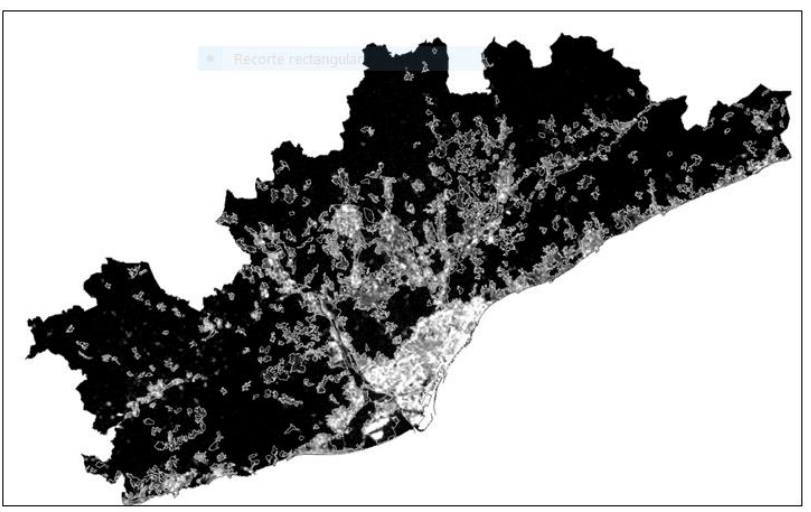

\begin{tabular}{|c|c|c|c|c|c|}
\hline \multicolumn{6}{|c|}{ Figure 8. Luojia 1-01 (MRB) } \\
\hline & & & \multicolumn{3}{|c|}{ Logistic Regression } \\
\hline & & & Rural & Urbanized & Total \\
\hline \multirow{9}{*}{ CLC } & \multirow{3}{*}{ Rural } & N. Pixels & 136747 & 19087 & 155834 \\
\hline & & $\%$ Row & $87,75 \%$ & $12,25 \%$ & $100,00 \%$ \\
\hline & & \% Column & $96,60 \%$ & $32,35 \%$ & $77,70 \%$ \\
\hline & \multirow{3}{*}{ Urbanized } & N. Pixels & 4814 & 39920 & 44734 \\
\hline & & \% Row & $10,76 \%$ & $89,24 \%$ & $100,00 \%$ \\
\hline & & \% Column & $3,40 \%$ & $67,65 \%$ & $22,30 \%$ \\
\hline & \multirow{3}{*}{ Total } & N. Pixels & 141561 & 59007 & 200568 \\
\hline & & $\%$ Row & $70,58 \%$ & $29,42 \%$ & $100,00 \%$ \\
\hline & & \% Column & $100,00 \%$ & $100,00 \%$ & $100,00 \%$ \\
\hline
\end{tabular}

Table 3. Urbanized Land (CLC) vs Luojia 1-01 (MRB)

\subsection{Shenzhen City}

Figures 9 to 10 show SNPP-VIIRS (2019) and Luojia 1-01 (2019) nighttime lights and the urbanized land according to FROM-GLC (2017) in Shenzhen City (Zheng, 2019). Tables 4 and 5 show the synthetic results (overall accuracy) of the iterative logistic regressions obtained for these sensors, since it has not been possible to obtain stable results with DMSP-OLS and Black Marble.

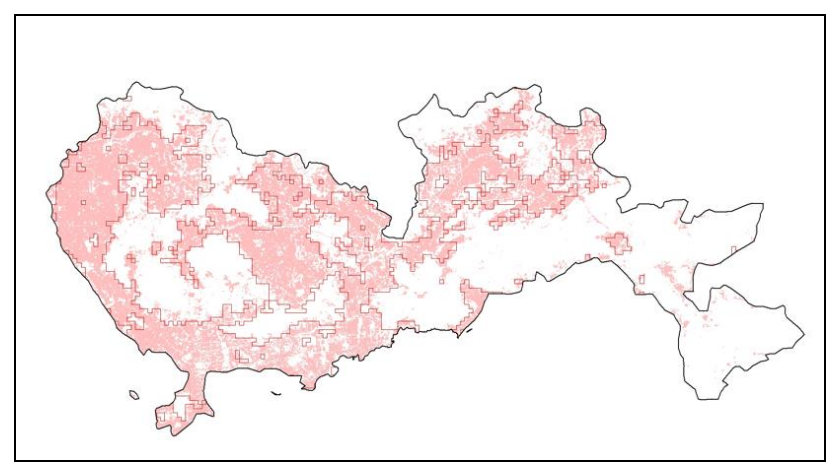

Figure 9. SNPP-VIIRS (Shenzhen)

\begin{tabular}{|ccc|ccc|}
\hline & & & \multicolumn{3}{|c|}{ Logistic Regression } \\
& & & Rural & Urbanized & Total \\
\hline \multirow{4}{*}{ Rural } & N. Pixels & 1128841 & 282395 & 1411236 \\
& & $\%$ Row & $79,99 \%$ & $20,01 \%$ & $100,00 \%$ \\
& & $\%$ Column & $85,27 \%$ & $31,28 \%$ & $63,38 \%$ \\
\cline { 3 - 6 } CLC & \multirow{3}{*}{ Urbanized } & N. Pixels & 195010 & 620047 & 815057 \\
& & $\%$ Row & $23,93 \%$ & $76,07 \%$ & $100,00 \%$ \\
& & \% Column & $14,73 \%$ & $68,68 \%$ & $36,60 \%$ \\
\cline { 3 - 6 } & \multirow{3}{*}{ Total } & N. Pixels & 1323851 & 902802 & 2226653 \\
& & $\%$ Row & $59,45 \%$ & $40,55 \%$ & $100,00 \%$ \\
& & \% Column & $100,00 \%$ & $100,00 \%$ & $100,00 \%$ \\
\hline
\end{tabular}

Table 4. Urbanized Land (FROM-GLC) vs SNPP-VIIRS (Shenzhen)

Logistic regression models developed with SNPP-VIIRS are shown in figure 9 and table 4 . Logistic regressions converge after 8 iterations, obtaining $79.99 \%$ of hits in rural land and $78.07 \%$ in urbanized land. Overall accuracy reaches $78.54 \%$. SNPP-VIIRS obtains medium-high results to identify the extent of artificialized covers of the MRB, as reflected by the Kappa coefficient (0.548).

In the case of Luojia 1-01 (figure 10 and table 5), logistic regressions converge after 7 iterations, obtaining $77.97 \%$ of successes in rural land and $82.91 \%$ in urbanized land, with an overall accuracy of $81.04 \%$. The degree of validity of the night light intensity obtained by means of the Luojia 1-01 sensor (with a 32-bit pixel depth and a spatial resolution of 130 meters) can be considered slightly high, as demonstrated by Kappa coefficient (0.606) achieved as well as the rest of the indicators used.

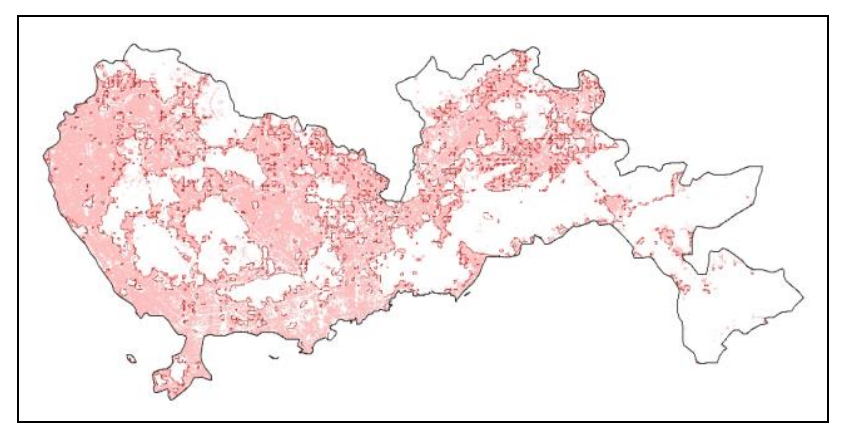

Figure 9. Luojia 1-01 (Shenzhen) 


\begin{tabular}{|ccc|ccc|}
\hline & & & \multicolumn{3}{|c|}{ Logistic Regression } \\
& & Rural & Urbanized & Total \\
\hline \multirow{6}{*}{ CLC } & \multirow{4}{*}{ Rural } & N. Pixels & 1128619 & 282617 & 1411236 \\
& & \% Row & $79,97 \%$ & $20,03 \%$ & $100,00 \%$ \\
& & \% Column & $89,01 \%$ & $29,48 \%$ & $63,38 \%$ \\
\cline { 2 - 6 } & \multirow{3}{*}{ Urbanized } & N. Pixels & 139363 & 676054 & 815417 \\
& & \% Row & $17,09 \%$ & $82,91 \%$ & $100,00 \%$ \\
& & \% Column & $10,99 \%$ & $70,52 \%$ & $36,62 \%$ \\
\cline { 2 - 6 } & \multirow{3}{*}{ Total } & N. Pixels & 1267982 & 958671 & 2226653 \\
& & \% Row & $56,95 \%$ & $43,05 \%$ & $100,00 \%$ \\
& & \% Column & $100,00 \%$ & $100,00 \%$ & $100,00 \%$ \\
\hline
\end{tabular}

Table 5. Urbanized Land (FROM-GLC) vs Luojia 1-01

(Shenzhen)

\section{CONCLUSIONS}

In this paper we have discussed the possibility of using different nighttime light remote sensors in order to analyze its usefulness in identifying urbanized land covers. The case studies have been Barcelona Metropolitan Region (Spain) and the city of Shenzhen (China).

In both cases, the results show clearly better performance of Luojia 1-01 in the identification of urbanized land. Tables 6 and 7 show the results of some indicators on the goodness of the adjustments for Barcelona and Shenzhen.

In the case of Barcelona, the Kappa value of the logistic regression models was 0.232 for DMSP-OLS, 0.420 for Black Marble, 0.578 for SNPP-VIIRS and 0.691 for Luojia 1-01. Overall accuracy varies from $54.89 \%$ for DMPS-OLS, to $75.25 \%$ for Black Marble, $82.52 \%$ for SNPP-VIIRS and 88.08 for Luojia 1-01.

\begin{tabular}{|lcccc|}
\hline & \% Rural & \% Urban & \% All Covers & Kappa \\
\hline DMSP-OLS* & 42.96 & 96.42 & 54.89 & 0.232 \\
Black Marble & 66.8 & 91.3 & 75.25 & 0.420 \\
SNPP-VIIRS & 80.9 & 88.3 & 82.52 & 0.578 \\
Juojia-01 & 87.8 & 89.2 & 88.08 & 0.691 \\
\hline
\end{tabular}

Table 6. Adjustment accuracy indicators (MRB)

Luojia 1-01 performed better in overall accuracy, and urbanized and rural land prediction and in the determination of land cover types: compact, industrial and dispersed fabrics. The new satellite is better at identifying the weak lights of the urban sprawl and determines rurban landscapes more efficiently, that is, rural land under urban development pressure.

In the case of Shenzhen, the Kappa value of the logistic regression models was 0.548 for SNPP-VIIRS and 0.606 for Luojia 1-01. Overall accuracy varies from $78.54 \%$ for SNPPVIIRS to 0.606 for Luojia 1-01.

\begin{tabular}{lcccc} 
& \% Rural & \% Urban & \% All Covers & Kappa \\
\hline SNPP-VIIRS & 77.99 & 77.07 & 78.54 & 0.548 \\
Loujia 1-01 & 79.97 & 82.91 & 81.05 & 0.606 \\
\hline
\end{tabular}

Table 7. Adjustment accuracy indicators (Shenzhen)

As in the case of the Metropolitan Region of Barcelona, in Shenzhen City Luojia 1-01 performed better in overall accuracy, and urbanized and rural land prediction and in the determination of land cover types. However, the results are worse than those obtained in the Metropolitan Region of Barcelona.

The lower goodness of fit of the regression models achieved in Shenzhen in relation with Barcelona is due to the lower accuracy of the FROM-GLC database comparing to Corine Land Cover (see Appendix). Particularly FROM-GLC does not accurately identify the urbanized land, considering only impervious surfaces. Urban green spaces are classified as rural covers, such as forest, grass or shrubs.

\section{ACKNOWLEDGEMENTS}

The authors would like to thank Zheng Qianhui for the collaboration provided in the construction of the logistic regression models in Shenzhen (China). Zheng Qianhui has been our student, in the Urban Management and Valuation line of the Advanced Master in Architecture (MBArch) developed at the School of Architecture of Barcelona (Technical University of Catalonia) in the academic year 2018-2019. His master's thesis (Zheng, 2019) has been directed by Josep Roca.

\section{REFERENCES}

Arellano, B. \& Roca, J., 2016. Landscapes impacted by light. The International Archives of the Photogrammetry, Remote Sensing and Spatial Information Sciences, Volume XLI-B8, 2016 XXIII ISPRS Congress, 12-19 July 2016, Prague, Czech Republic.

Arellano, B. and Roca, J., 2018. The urbanization impact in China: a prospective model (1992-2025). Proc. SPIE, 10767, Remote Sensing and Modeling of Ecosystems for Sustainability XV, 107670A (18 September 2018).

Corine Land Cover, 2019. CLC V20 Lineage, reduced version. https://land.copernicus.eu/user-corner/technical-library/clc-andclcc-release-lineage

Elvidge, C.D., Imhoff, M.L., Baugh, K.E., Hobson, V.R., Nelson, I., Safran, J., Dietz, J.B. and Tuttle, B.T., 2001. Nighttime lights of the world: 1994-1995. ISPRS Journal of Photogrammetry \& Remote Sensing, 56, 81-99 (2001).

Elvidge, C.D., Baugh, K., Zhizhin, M. and Hsu, F. C., 2013. Why VIIRS data are superior to DMSP for mapping night-time lights. Proceedings of the Asia-Pacific Advanced Network, 35, 62-69 (2013).

Florida, R., Gulden, T. and Mellander, C., 2008. The rise of the mega-region. Cambridge Journal of Regions, Economy and Society, 1 (3), 459-476 (2008).

Jiang, W., He, G., Long, T., Guo, H., Yin, R., Leng, W., Liu, H., and Wang, G., 2018. Potentiality of Using Luojia 1-01 Nighttime Light Imagery to Investigate Artificial Light Pollution. Sensors 18, 2900, 1-15 (2018).

NASA Earth Observatory, 2012. Night Lights 2012 - The Black Marble. https://earthobservatory.nasa.gov/images/79803/nightlights-2012-the-black-marble 
United States Census Bureau, 2010. Census Urban and Rural Classification and Urban Area Criteria. https:// www.census.gov/geo/reference/ua/urban-rural-2010.html.

Su, Y., Chen, X., Wang, C., Zhang, H., Liao, J., Ye, Y. \& Wang, C., 2015. A new method for extracting built-up urban areas using DMSP-OLS nighttime stable lights: A case study in the Pearl River Delta, southern China. GIScience \& Remote Sensing, vol. 52, no. 2, pp. 218-238.

Yu, B. et al., 2018. Urban Built-Up Area Extraction From LogTransformed NPP-VIIRS Nighttime Light Composite Data. IEEE Geoscience and Remote Sensing Letters, vol. 15, no. 8, pp. 1279-1283, Aug. 2018.

Zheng, Q., 2019. La re-extracción de áreas urbanizadas basada en las imágenes de luces nocturnas de alta resolución. Caso de estudio: Shenzhen, Guangdong, China. https://upcommons.upc.edu/handle/2117/179573.

Zhou, Y., Smith, S.J., Elvidge, C.D., Zhao, K., Thomson, A. \& Imhoff, M., 2014. A cluster-based method to map urban area from DMSP/OLS nightlights. Remote Sensing of Environment, vol. 147, pp. 173-185, May 2014.

\section{APPENDIX}

\section{Metropolitan Region of Barcelona}

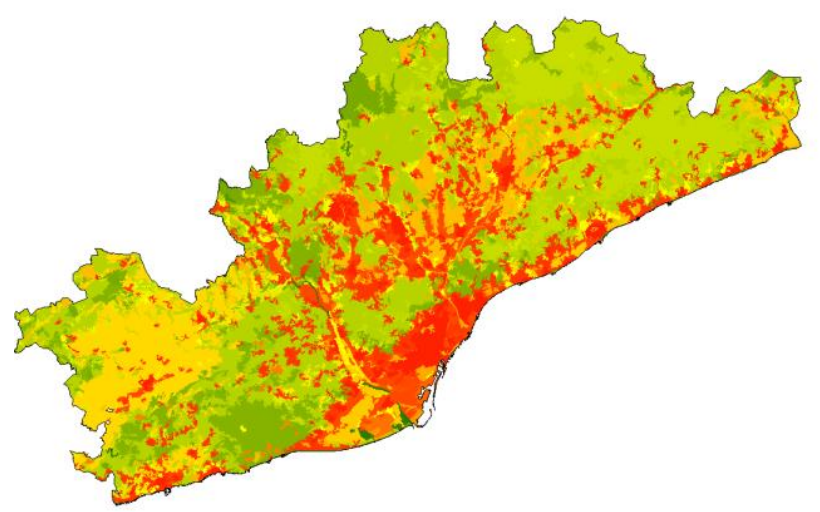

Corine Land Cover (RMB)

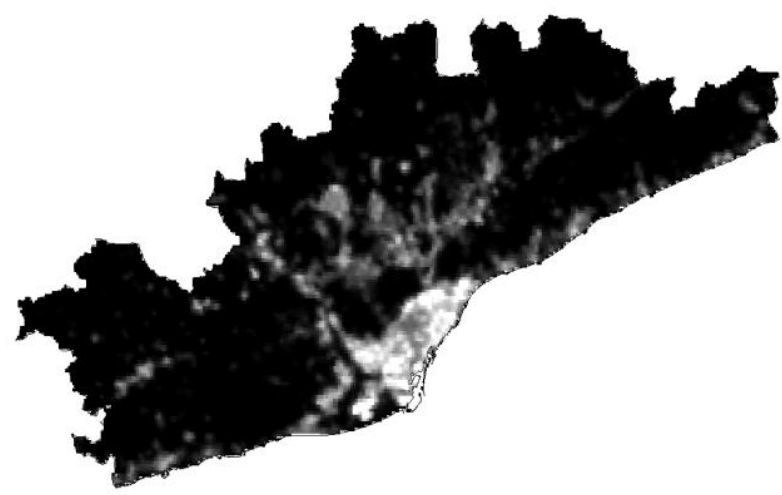

SNPP-VIIRS (RMB)

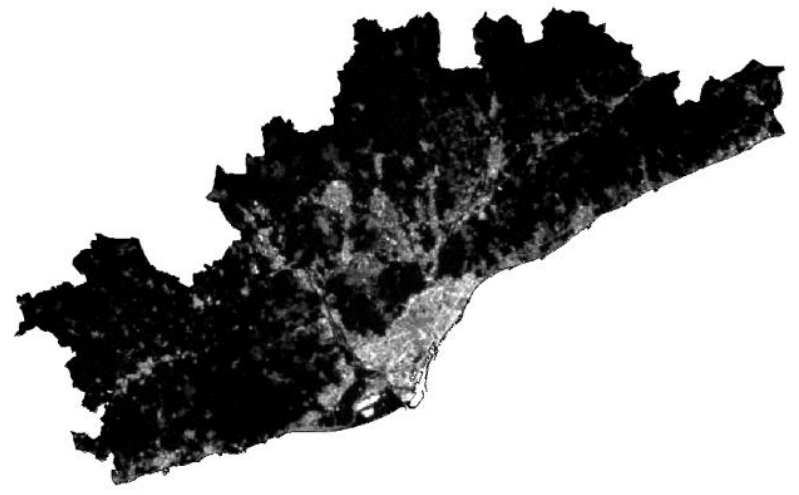

Luojia 1-01 (RMB)

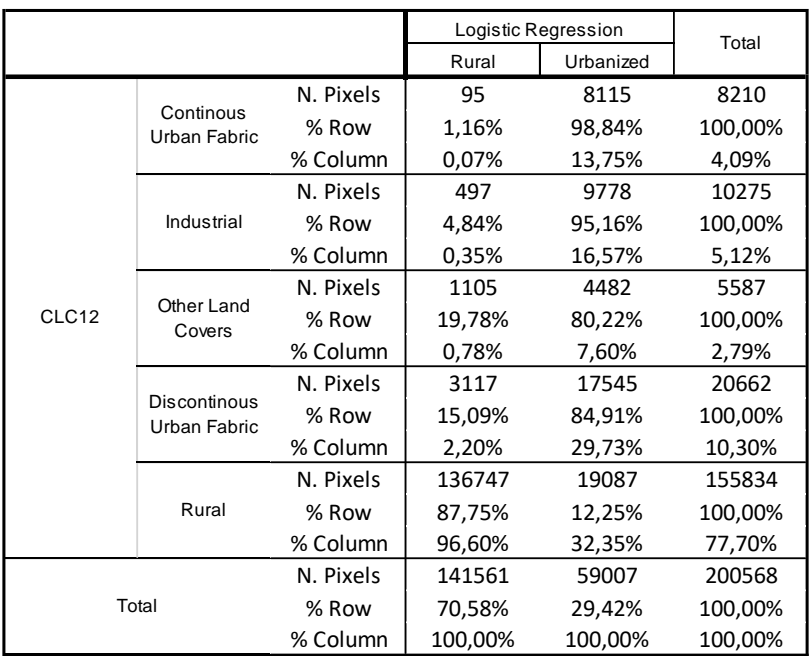

Overall Accuracy by Land Covers (RMB)

\section{Shenzhen}

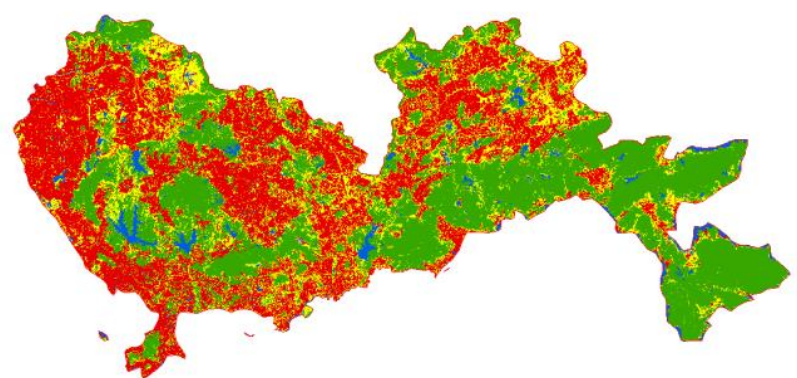

FROM-GLC

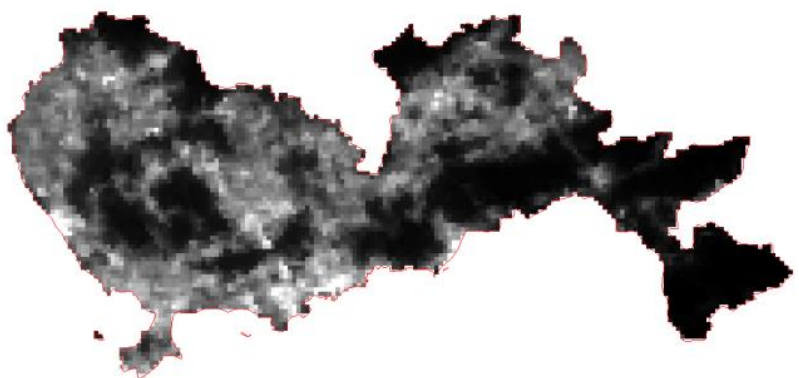

SNPP-VIIRS 


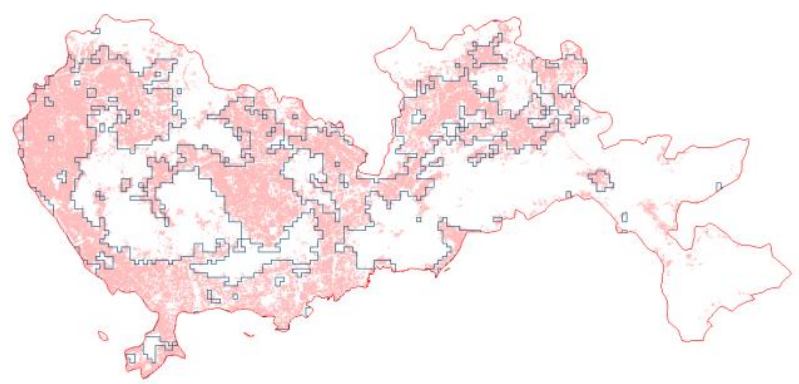

Urban Classification SNPP-VIIRS

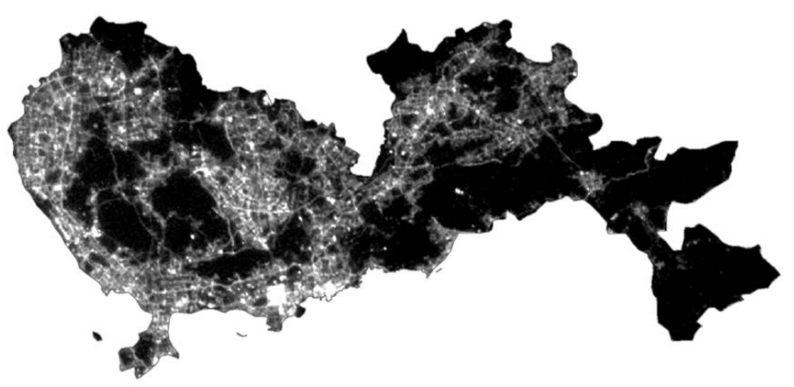

Luojia 1-01

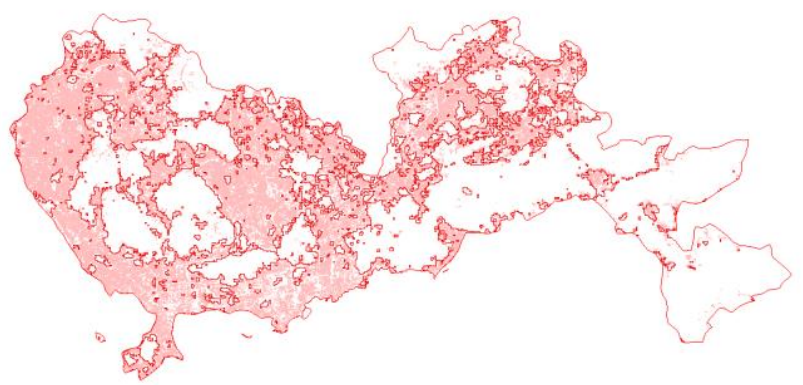

Urban Classification Luojia 1-01 